Article

\title{
Cyclization of Hydrazones of 2-Acetyl-1-naphthol and 1-Acetyl- 2-naphthol with Triphosgene. Synthesis of Spiro Naphthoxazine Dimers
}

\author{
Abdullah Saad Al-Bogami ${ }^{1}$, Abdullah Mohammed Al-Majid ${ }^{1}$, Mohammed Ali Al-Saad ${ }^{1}$, \\ Ahmed Amine Mousa ${ }^{1}$, Sara Abdullah Al-Mazroa ${ }^{2}$ and Hamad Zaid Alkhathlan ${ }^{1}{ }^{\text {** }}$ \\ 1 Department of Chemistry, King Saud University, P. O. Box 2455, Riyadh 11451, Saudi Arabia \\ 2 Chemistry Department, Girls College of Education, Riyadh Girls University, Riyadh, Saudi Arabia \\ * Author to whom correspondence should be addressed; E-mail: khathlan@ksu.edu.sa; \\ Tel: +966-1-4675910; Fax: +966-1-4675992
}

Received: 25 April 2009; in revised form: 15 May 2009 / Accepted: 5 June 2009 /

Published: 12 June 2009

\begin{abstract}
Cyclization of hydrazones derived from 2-acetyl-1-naphthol and 1-acetyl-2naphthol with triphosgene gave naphtho[1,2-e]-1,3-oxazines, naphtho[2,1-e]-1,3-oxazines or their spiro dimers depending on the molar ratio of triphosgene used for the cyclization.
\end{abstract}

Keywords: 2-acetyl-1-naphthol; 1-acetyl-2-naphthol; triphosgene; naphthoxazine, spiro naphthoxazine

\section{Introduction}

Triphosgene (bis(trichloromethyl)carbonate) has been repeatedly used in the literature for the construction of a variety of heterocyclic systems. It holds an advantage over other similar reagents, such as phosgene and diphosgene, of being a safe and easy to handle solid. Examples of important heterocyclic systems prepared using this reagent include benzothiadiazepines [1], quinazolines [2], diazolidines [3], imidiazolidines [4] and azetidines [5]. We have recently reported the use of triphosgene in the cyclization of hydrazones and Schiff bases of 2-hydroxy- and 2aminoacetophenones to give 1,3-benzoxazines [6,7], spiro 1,3-benzoxazine dimmers [8], quinazolines and spiro quinazoline dimers [9]. 
We would like to report here results obtained from the cyclization of hydrazones of 2-acetyl-1naphthol and 1-acetyl-2-naphthol with triphosgene.

\section{Results and Discussion}

Hydrazones of 2-acetyl-1-naphthol and 1-acetyl-2-naphthol were obtained in very good yields from the reaction of these compounds with aromatic hydrazines (Scheme 1, Table 1).

Scheme 1. Synthesis of hydrazones of 2-acetyl-1-naphthol and 1-acetyl-2-naphthol.

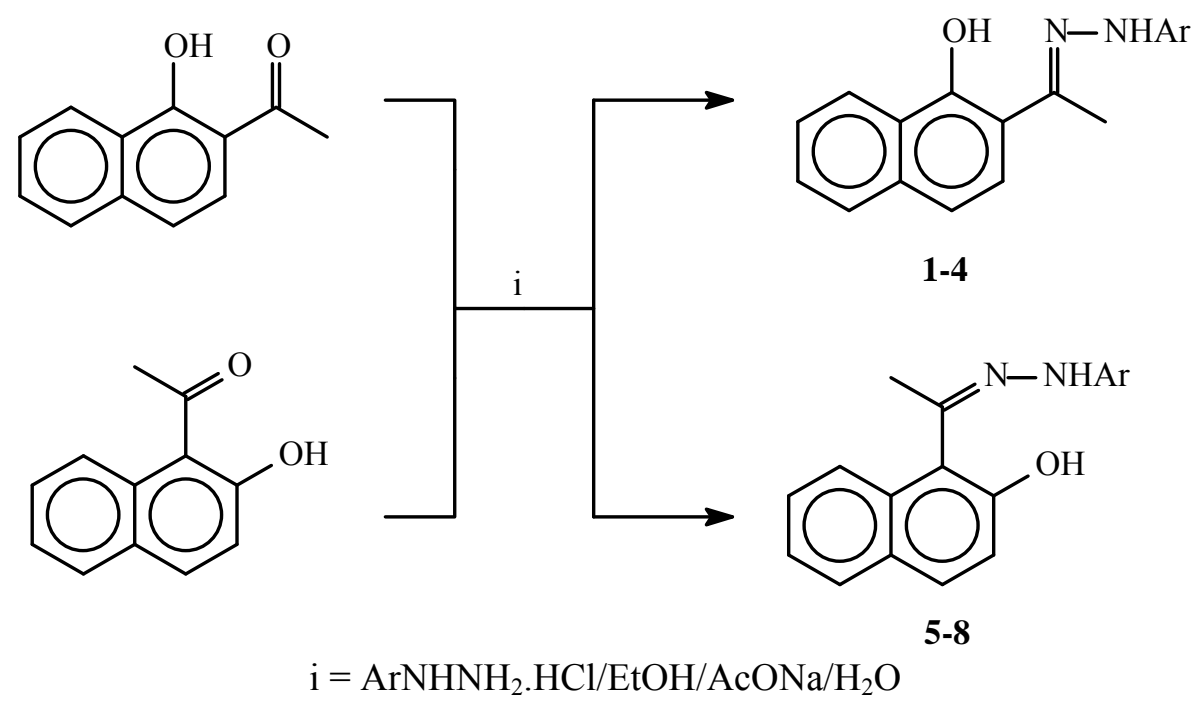

Table 1. Hydrazones 1-8.

\begin{tabular}{clc}
\hline Compound & \multicolumn{1}{c}{$\mathrm{Ar}$} & Yield (\%) \\
\hline $\mathbf{1}$ & Phenyl & 92 \\
$\mathbf{2}$ & $4-\mathrm{ClC}_{6} \mathrm{H}_{4}$ & 82 \\
$\mathbf{3}$ & $4-\mathrm{BrC}_{6} \mathrm{H}_{4}$ & 79 \\
$\mathbf{4}$ & $4-\mathrm{CH}_{3} \mathrm{C}_{6} \mathrm{H}_{4}$ & 91 \\
$\mathbf{5}$ & $\mathrm{Phenyl}$ & 75 \\
$\mathbf{6}$ & $4-\mathrm{ClC}_{6} \mathrm{H}_{4}$ & 80 \\
$\mathbf{7}$ & $4-\mathrm{BrC}_{6} \mathrm{H}_{4}$ & 68 \\
$\mathbf{8}$ & $4-\mathrm{CH}_{3} \mathrm{C}_{6} \mathrm{H}_{4}$ & 68 \\
\hline
\end{tabular}

The hydrazones 1-8 show in the their IR spectra absorbances for the $\mathrm{C}=\mathrm{N}$ group in the 1,614$1,636 \mathrm{~cm}^{-1}$ range and for the $\mathrm{OH}$ and $\mathrm{NH}$ groups at about $3,400 \mathrm{~cm}^{-1}$ and $3,350 \mathrm{~cm}^{-1}$, respectively. The ${ }^{1} \mathrm{H}-\mathrm{NMR}$ spectra of compounds 1-8 show in each case two distinct doublets which have been assigned to the $\mathrm{H}_{3}$ and $\mathrm{H}_{4}$ positions. In the spectra of 1-4 these two doublets appear at $\delta$ 7.04-7.16 and $\delta$ 7.68-7.76. The lower field signal in this case was assigned to $\mathrm{H}_{3}$ as it is deshielded by the hydrazone moiety. In contrast, for compounds 5-8, where the two doublets appear at $\delta 6.80-6.90$ and $\delta 7.63-7.66$ the signal at the higher field was assigned to $\mathrm{H}_{3}$ as in this case this position is shielded by the $\mathrm{OH}$ group. It is also worth mentioning here that in the ${ }^{13} \mathrm{C}-\mathrm{NMR}$ spectra of the above compounds, the methyl group of the hydrazones of the 2-acetyl derivatives 1-4 absorbs at higher fields $(\delta 12.22-13.04)$ 
than those of the 1-acetyl derivatives 5-8 $(\delta$ 18.29-18.55). The rest of the spectral data is shown in the Experimental section. Treatment of the hydrazones 1-8 with triphosgene gave the 4-methylenenaphthoxazines 9-16 or the spiro naphthoxazine dimers 17-20, depending on the molar ratio of triphosgene used in the cyclizations (Schemes 2 and 3, Table 2).

Scheme 2. Reaction of hydrazones 1-4 with triphosgene.
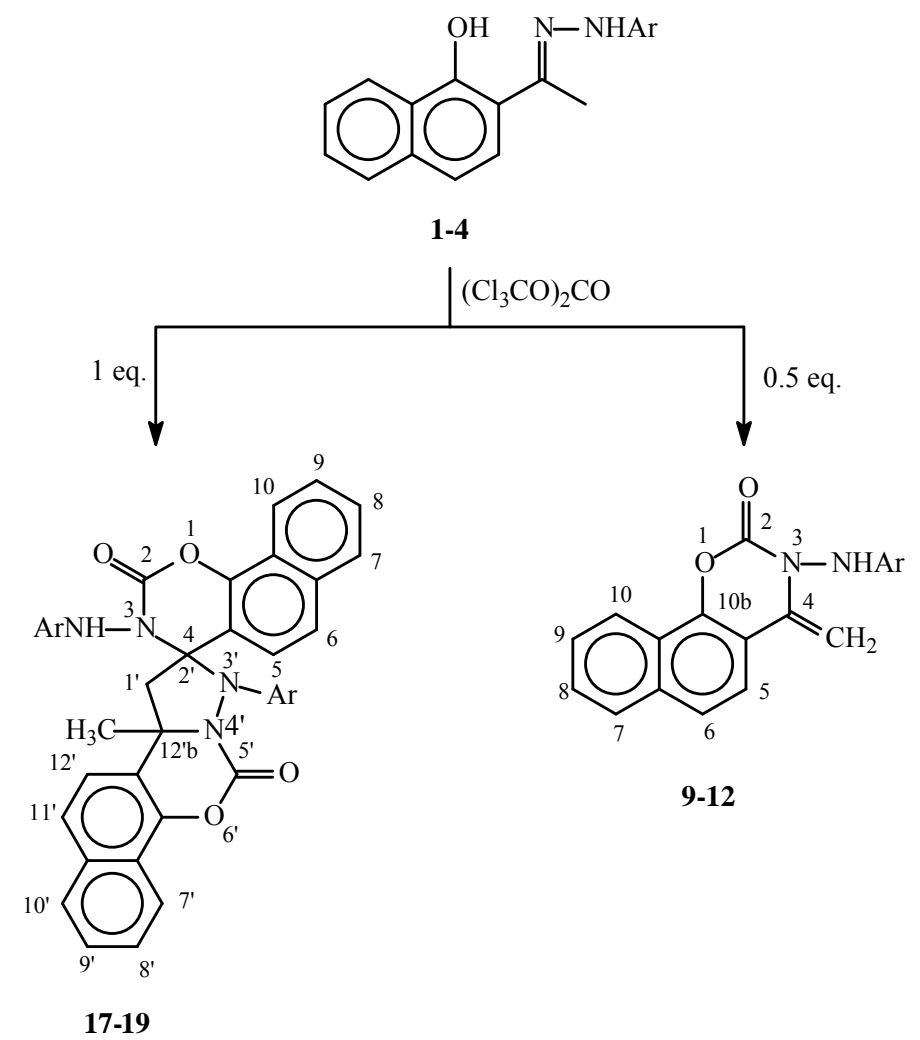

Scheme 3. Reaction of hydrazones 5-8 with triphosgene.

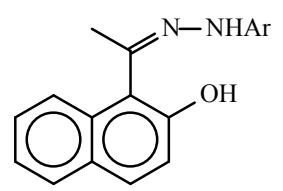

5-8
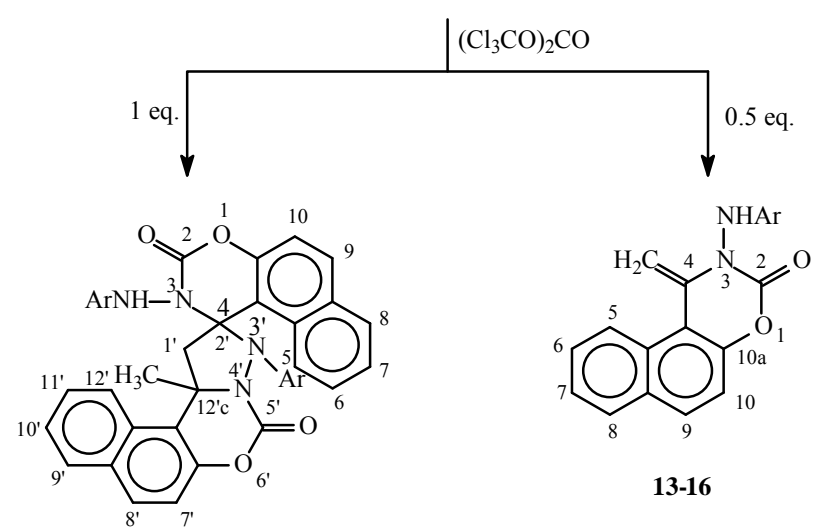
Table 2. 4-Methylenenaphthoxazines 9-16 and spiro naphthoxazine dimmers 17-20.

\begin{tabular}{|c|c|c|}
\hline Compound & $\mathrm{Ar}$ & Yield $(\%)$ \\
\hline 9 & Phenyl & 69 \\
\hline 10 & $4-\mathrm{ClC}_{6} \mathrm{H}_{4}$ & 74 \\
\hline 11 & 4- $\mathrm{BrC}_{6} \mathrm{H}_{4}$ & 71 \\
\hline 12 & $4-\mathrm{CH}_{3} \mathrm{C}_{6} \mathrm{H}_{4}$ & 73 \\
\hline 13 & Phenyl & 62 \\
\hline 14 & $4-\mathrm{ClC}_{6} \mathrm{H}_{4}$ & 61 \\
\hline 15 & 4- $\mathrm{BrC}_{6} \mathrm{H}_{4}$ & 64 \\
\hline 16 & $4-\mathrm{CH}_{3} \mathrm{C}_{6} \mathrm{H}_{4}$ & 60 \\
\hline 17 & Phenyl & 69 \\
\hline 18 & $4-\mathrm{ClC}_{6} \mathrm{H}_{4}$ & 72 \\
\hline 19 & 4- $\mathrm{BrC}_{6} \mathrm{H}_{4}$ & 71 \\
\hline 20 & $4-\mathrm{CH}_{3} \mathrm{C}_{6} \mathrm{H}_{4}$ & 55 \\
\hline
\end{tabular}

These results are in agreement with our previous reports on the reaction of hydrazones of 2-hydroxyacetophenones with triphosgene. It was observed then that the use of 0.5 equivalents of triphosgene gave the 4-methylene-1,3-benzoxazines, while the spiro 1,3-benzoxazine dimers were obtained when 1.0 equivalent of triphosgene was used [8]. The structures of those compounds were established based on X-ray crystal structure and spectroscopic studies [8,9]. As a result, we are showing here partial spectroscopic data for compounds 9-16 (Tables 3 and 4) and 17-20 (Table 5). In these tables, signals that can be easily attributed to structurally related positions, and with significance to the elucidation of the structures of the new compounds are shown for matter of comparison, while the complete data is given in the experimental section.

Table 3. Partial IR and NMR data for compounds 9-16.

\begin{tabular}{|c|c|c|c|c|}
\hline \multirow{2}{*}{ Compound } & \multicolumn{2}{|c|}{ IR $\left(\mathrm{cm}^{-1}\right)$} & \multicolumn{2}{|c|}{${ }^{1}$ H-NMR (ppm) } \\
\hline & $\mathrm{C}=\mathrm{O}$ & $=\mathrm{CH}_{2}$ & $=\mathrm{CH}_{2}$ & Aromatic \\
\hline \multirow[t]{3}{*}{9} & 1,727 & 1,620 & $5.02(\mathrm{~d}, J=2.2 \mathrm{~Hz}, 1 \mathrm{H})$ & $6.80\left(\mathrm{~d}, J=8.1 \mathrm{~Hz}, 2 \mathrm{H}, \mathrm{H}_{2,6, \mathrm{Ar}}\right)$ \\
\hline & & & $5.13(\mathrm{~d}, J=2.2 \mathrm{~Hz}, 1 \mathrm{H})$ & $7.61\left(\mathrm{~d}, J=8.8 \mathrm{~Hz}, 1 \mathrm{H}, \mathrm{H}_{6}\right)$ \\
\hline & & & & $7.66\left(\mathrm{~d}, J=8.8 \mathrm{~Hz}, 1 \mathrm{H}, \mathrm{H}_{5}\right)$ \\
\hline \multirow[t]{3}{*}{10} & 1,732 & 1,621 & $5.02(\mathrm{~d}, J=2.2 \mathrm{~Hz}, 1 \mathrm{H})$ & $6.78\left(\mathrm{~d}, J=9.0 \mathrm{~Hz}, 2 \mathrm{H}, \mathrm{H}_{2,6, \mathrm{Ar}}\right)$ \\
\hline & & & $5.09(\mathrm{~d}, J=2.2 \mathrm{~Hz}, 1 \mathrm{H})$ & $7.23\left(\mathrm{~d}, J=9.0 \mathrm{~Hz}, 2 \mathrm{H}, \mathrm{H}_{3,5, \mathrm{Ar}}\right)$ \\
\hline & & & & $7.67\left(\mathrm{~d}, J=8.8 \mathrm{~Hz}, 1 \mathrm{H}, \mathrm{H}_{5}\right)$ \\
\hline \multirow[t]{2}{*}{11} & 1,741 & 1,621 & $4.96(\mathrm{~d}, J=2.0 \mathrm{~Hz}, 1 \mathrm{H})$ & $6.73\left(\mathrm{~d}, J=8.8 \mathrm{~Hz}, 2 \mathrm{H}, \mathrm{H}_{2,6, \mathrm{Ar})}\right.$ \\
\hline & & & $5.01(\mathrm{~d}, J=2.0 \mathrm{~Hz}, 1 \mathrm{H})$ & $7.24\left(\mathrm{~d}, J=8.8 \mathrm{~Hz}, 2 \mathrm{H}, \mathrm{H}_{3,5, \mathrm{Ar}}\right)$ \\
\hline \multirow[t]{4}{*}{12} & 1,737 & 1,612 & $4.92(\mathrm{~d}, J=2.0 \mathrm{~Hz}, 1 \mathrm{H})$ & $6.71\left(\mathrm{~d}, J=8.1 \mathrm{~Hz}, 2 \mathrm{H}, \mathrm{H}_{2,6, \mathrm{Ar}}\right)$ \\
\hline & & & $5.14(\mathrm{~d}, J=2.0 \mathrm{~Hz}, 1 \mathrm{H})$ & $7.02\left(\mathrm{~d}, J=8.1 \mathrm{~Hz}, 2 \mathrm{H}, \mathrm{H}_{3,5, \mathrm{Ar}}\right)$ \\
\hline & & & & $7.84\left(\mathrm{~d}, J=8.8 \mathrm{~Hz}, 1 \mathrm{H}, \mathrm{H}_{6}\right)$ \\
\hline & & & & $7.91\left(\mathrm{~d}, J=8.8 \mathrm{~Hz}, 1 \mathrm{H}, \mathrm{H}_{5}\right)$ \\
\hline \multirow[t]{2}{*}{13} & 1,725 & 1,620 & $5.04(\mathrm{~d}, J=2.2 \mathrm{~Hz}, 1 \mathrm{H})$ & $6.97\left(\mathrm{~d}, J=8.8 \mathrm{~Hz}, 2 \mathrm{H}, \mathrm{H}_{2,6, \mathrm{Ar}}\right)$ \\
\hline & & & $5.15(\mathrm{~d}, J=2.2 \mathrm{~Hz}, 1 \mathrm{H})$ & $7.67\left(\mathrm{~d}, J=8.1 \mathrm{~Hz}, 1 \mathrm{H}, \mathrm{H}_{9}\right)$ \\
\hline
\end{tabular}


Table 3. Cont.

\begin{tabular}{|c|c|c|c|c|}
\hline 14 & 1,732 & 1,620 & $\begin{array}{l}5.05(\mathrm{~d}, J=1.5 \mathrm{~Hz}, 1 \mathrm{H}) \\
5.15(\mathrm{~d}, J=1.5 \mathrm{~Hz}, 1 \mathrm{H})\end{array}$ & $\begin{array}{c}6.84\left(\mathrm{~d}, J=8.8 \mathrm{~Hz}, 2 \mathrm{H}, \mathrm{H}_{2,6, \mathrm{Ar}}\right) \\
7.23\left(\mathrm{~d}, J=8.8 \mathrm{~Hz}, 2 \mathrm{H}, \mathrm{H}_{3,5, \mathrm{Ar}}\right) \\
7.70\left(\mathrm{~d}, J=8.1 \mathrm{~Hz}, 1 \mathrm{H}, \mathrm{H}_{9}\right)\end{array}$ \\
\hline 15 & 1,733 & 1,616 & $\begin{array}{l}5.05(\mathrm{~d}, J=2.2 \mathrm{~Hz}, 1 \mathrm{H}) \\
5.14(\mathrm{~d}, J=2.2 \mathrm{~Hz}, 1 \mathrm{H})\end{array}$ & $\begin{array}{l}6.90\left(\mathrm{~d}, J=8.0 \mathrm{~Hz}, 2 \mathrm{H}, \mathrm{H}_{2,6, \mathrm{Ar}}\right) \\
7.29\left(\mathrm{~d}, J=8.0 \mathrm{~Hz}, 2 \mathrm{H}, \mathrm{H}_{3,5, \mathrm{Ar}}\right)\end{array}$ \\
\hline 16 & 1,741 & 1,615 & $\begin{array}{l}5.04(\mathrm{~d}, J=2.2 \mathrm{~Hz}, 1 \mathrm{H}) \\
5.14(\mathrm{~d}, J=2.2 \mathrm{~Hz}, 1 \mathrm{H})\end{array}$ & $\begin{array}{c}6.81\left(\mathrm{~d}, J=8.8 \mathrm{~Hz}, 2 \mathrm{H}, \mathrm{H}_{2,6, \mathrm{Ar}}\right) \\
7.08\left(\mathrm{~d}, J=8.8 \mathrm{~Hz}, 2 \mathrm{H}, \mathrm{H}_{3,5, \mathrm{Ar}}\right) \\
7.66\left(\mathrm{~d}, J=8.8 \mathrm{~Hz}, 1 \mathrm{H}, \mathrm{H}_{9}\right)\end{array}$ \\
\hline
\end{tabular}

Table 4. Partial ${ }^{13} \mathrm{C}-\mathrm{NMR}$ data for 9-16.

\begin{tabular}{ccccccccc}
\hline & $\mathbf{9}$ & $\mathbf{1 0}$ & $\mathbf{1 1}$ & $\mathbf{1 2}$ & $\mathbf{1 3}$ & $\mathbf{1 4}$ & $\mathbf{1 5}$ & $\mathbf{1 6}$ \\
\hline $\mathrm{C}_{1, \mathrm{Ar}}$ & 143.05 & 143.46 & 143.35 & 143.34 & 143.53 & 143.48 & 143.53 & 142.88 \\
$\mathrm{C}_{2,6, \mathrm{Ar}}$ & 113.03 & 115.70 & 114.89 & 113.07 & 113.97 & 115.33 & 113.96 & 114.12 \\
$\mathrm{C}_{3,5, \mathrm{Ar}}$ & 129.30 & 132.33 & 131.99 & 130.03 & 129.45 & 129.44 & 129.47 & 129.96 \\
$\mathrm{C}_{2}$ & 148.05 & 147.99 & 147.35 & 147.21 & 148.07 & 148.01 & 148.08 & 148.10 \\
$\mathrm{C}_{4}$ & 138.69 & 138.26 & 138.54 & 138.99 & 138.46 & 138.30 & 138.45 & 138.48 \\
$=\mathrm{CH}_{2}$ & 88.60 & 88.55 & 88.58 & 88.97 & 88.53 & 88.53 & 88.54 & 88.50 \\
$\mathrm{C}_{5}\left(\mathrm{C}_{10}\right)$ & 122.93 & 121.48 & 121.52 & 121.50 & $(120.33)$ & $(120.25)$ & $(120.33)$ & $(120.34)$ \\
$\mathrm{C}_{6}\left(\mathrm{C}_{9}\right)$ & 120.69 & 120.24 & 120.73 & 121.05 & $(128.12)$ & $(128.23)$ & $(128.13)$ & $(128.10)$ \\
$\mathrm{C}_{10 \mathrm{~b}}\left(\mathrm{C}_{10 \mathrm{a}}\right)$ & 146.16 & 144.45 & 145.60 & 144.14 & $(145.29)$ & $(143.95)$ & $(145.29)$ & $(143.53)$ \\
$\mathrm{C}_{2}$ & 148.05 & 147.99 & 147.35 & 147.21 & 148.07 & 148.01 & 148.08 & 148.10 \\
\hline
\end{tabular}

Table 5. Partial ${ }^{1} \mathrm{H}$ and ${ }^{13} \mathrm{C}-\mathrm{NMR}$ data for the spiro compounds 17- 20.

\begin{tabular}{|c|c|c|c|c|c|c|c|}
\hline & \multicolumn{2}{|r|}{${ }^{1} \mathrm{H}$} & \multicolumn{5}{|c|}{${ }^{13} \mathrm{C}$} \\
\hline & $\mathrm{CH}_{3}$ & $\mathrm{C}_{1}$ & $\mathrm{CH}_{3}$ & $\mathrm{C}_{1}$ & $\mathrm{C}_{2}$ & $\mathrm{C}_{12 \mathrm{~b}}$ & $\mathrm{C}=\mathrm{O}$ \\
\hline \multirow[t]{2}{*}{17} & 2.12 & $3.58(\mathrm{~d}, J=14.70 \mathrm{~Hz}, 1 \mathrm{H})$ & 32.15 & 59.65 & 85.55 & 66.06 & 149.24 \\
\hline & & $3.69(\mathrm{~d}, J=14.70 \mathrm{~Hz}, 1 \mathrm{H})$ & & & & & 149.90 \\
\hline \multirow[t]{2}{*}{18} & 2.10 & $3.49(\mathrm{~d}, J=14.70 \mathrm{~Hz}, 1 \mathrm{H})$ & 32.08 & 60.07 & 85.47 & 66.05 & 149.01 \\
\hline & & $3.60(\mathrm{~d}, J=14.70 \mathrm{~Hz}, 1 \mathrm{H})$ & & & & & 149.07 \\
\hline \multirow[t]{2}{*}{19} & 2.09 & $3.44(\mathrm{~d}, J=14.70 \mathrm{~Hz}, 1 \mathrm{H})$ & 32.04 & 60.11 & 85.41 & 65.96 & 148.95 \\
\hline & & $3.54(\mathrm{~d}, J=14.70 \mathrm{~Hz}, 1 \mathrm{H})$ & & & & & 149.15 \\
\hline \multirow[t]{2}{*}{20} & 2.51 & $3.41(\mathrm{~d}, J=14.65 \mathrm{~Hz}, 1 \mathrm{H})$ & 17.20 & 27.87 & 97.68 & $(88.06)^{*}$ & 148.60 \\
\hline & & $3.54(\mathrm{~d}, J=14.65 \mathrm{~Hz}, 1 \mathrm{H})$ & & & & & 149.01 \\
\hline
\end{tabular}

$*\left(\mathrm{C}_{12^{\prime} \mathrm{c}}\right)$.

It can be seen from Table 3 that there is a noticeable difference in the ${ }^{13} \mathrm{C}$-NMR spectra of 17-19 compared with 20. In the latter compound the $\mathrm{CH}_{3}$ group and position $\mathrm{C}_{1}$ absorb at higher fields than those in 17-19, while positions $C_{2}$ and $C_{12}{ }^{\prime}$ absorb at lower fields than positions $C_{2^{\prime}}$ and $C_{12}$ 'b in 17-19. This might be speculated as a result of the orientation of the aromatic rings in respect to these positions, which needs further studies. In addition, the above results for compounds 17-19 are in close 
resemblance to these reported for the spiro 1,3-benzoxazine dimmers which might suggest that they have similar steric environment for the 1,3-benzoxazines[8].

The EIMS spectra of the spiro compounds 17-20 did not show the expected molecular ions. They appear to fragment at the spiro junction to give fragments at $\mathrm{m} / \mathrm{z}=\mathrm{M}^{+}-\mathrm{ArN}_{2} \mathrm{CO}$ and $\mathrm{m} / \mathrm{z}=\mathrm{M}^{+}-\mathrm{ArN}_{2} \mathrm{CO}_{2}$. For example, compound 18 shows in its EIMS spectra fragment peaks at $\mathrm{m} / \mathrm{z}$ 505 and 489 , arising from the loss of $\mathrm{ClC}_{6} \mathrm{H}_{4} \mathrm{~N}_{2} \mathrm{CO}$ and $\mathrm{ClC}_{6} \mathrm{H}_{4} \mathrm{~N}_{2} \mathrm{CO}_{2}$, respectively. This type of fragmentation was previously observed with analogous spiro compounds[8,9].

Finally, it is worth mentioning here that although the formation of the 4-methylene-naphthoxazines 9-16 would be expected to proceed via a similar reaction mechanism to that previously reported for the cyclization of hydrazones of 2-hydroxyacetophenone and acetophenone [6,10], the mechanism for formation of the spiro naphthoxazine dimmers is not clear to us with regards to at what stage does the dimerization occurs to form the final spiro naphthoxazines.

Scheme 4. Proposed mechanism for the formation of the spiro compounds 17-20.

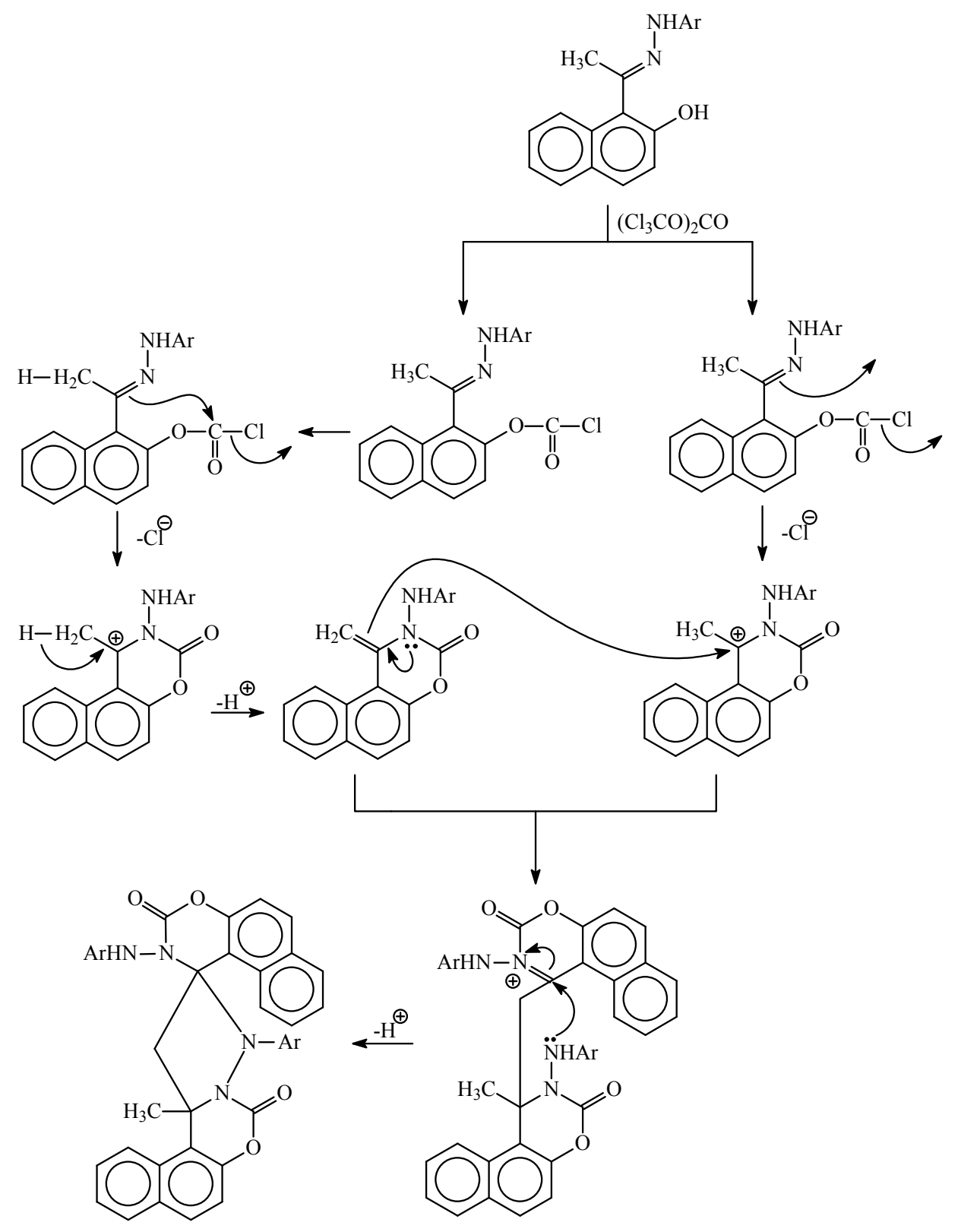


Scheme 4. Cont.

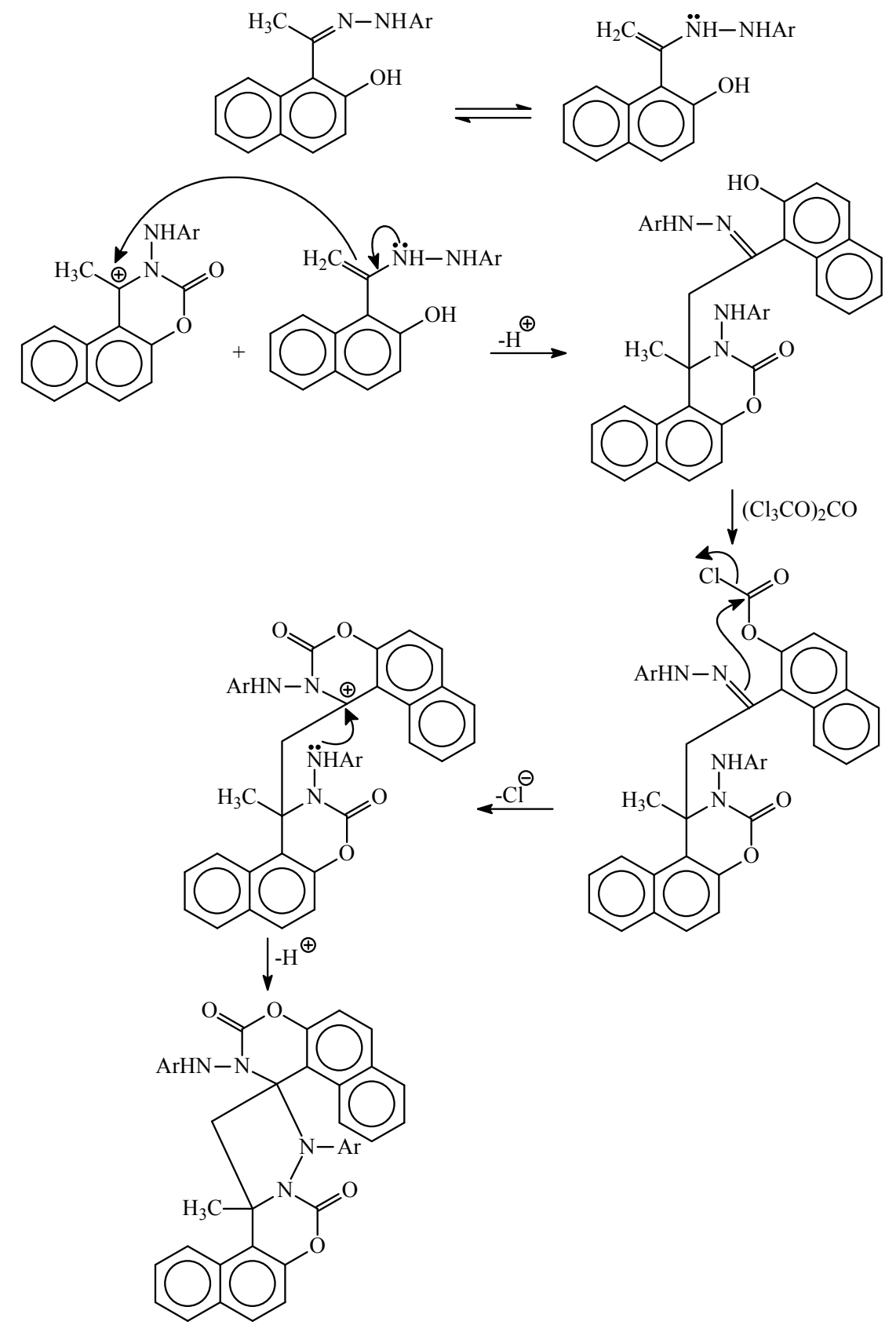

The use of 1.0 equivalents of triphosgene is necessary to form the latter products because in its reactions it gives an equivalent of three moles of phosgene $\left(\mathrm{COCl}_{2}\right)$, so the formation of naphthoxazines (where one $\mathrm{CO}$ group is introduced) would require only 0.5 equivalent of triphosgene (1.5 equivalent of $\mathrm{COCl}_{2}$, a slight excess is usually used in these kinds of cyclizations) while the spiro dimers (where two CO groups are introduced) would require double this amount. In Scheme 4, a speculative mechanism for the formation of the spiro products is shown with two pathways; in the first one the dimerization occurs after the formation of the two naphthoxazine nuclei, while in the second one the dimerization takes place before the formation of the second naphthoxazine ring. 


\section{Experimental}

Melting points are uncorrected. IR spectra were recorded on a Perkin-Elmer 883 spectrophotometer as $\mathrm{KBr}$ pellets and expressed as $v$ in $\mathrm{cm}^{-1}$. NMR spectra were recorded on JEOL ECP 400 (400 MHz) in $\mathrm{CDCl}_{3}$ and chemical shifts are expressed as $\delta$ in ppm. MS spectra were recorded on Shimadzu QP 5050A GC/MS system.

2-Acetyl-1-naphthol phenylhydrazone (1): To a solution of 2-acetyl-1-naphthol (2.0 g, $10.8 \mathrm{mmol})$ in ethanol $(50 \mathrm{~mL})$ was added a solution of phenylhydrazine hydrochloride $(1.9 \mathrm{~g}, 12.9 \mathrm{mmol})$ and sodium acetate $(1.0 \mathrm{~g}, 12.2 \mathrm{mmol})$ in a mixture of ethanol $(30 \mathrm{~mL})$ and water $(10 \mathrm{~mL})$. The above solution was refluxed for 5 hours and then evaporated under vacuum. The resulting solid was washed with water $(50 \mathrm{~mL})$ and then recrystallized from ethanol-chloroform (8:2). Yield 92\%, red solid, mp $162^{\circ} \mathrm{C}$; IR: 3,410 (OH), 3,334 (NH), 1,620 (C=N); ${ }^{1} \mathrm{H}-\mathrm{NMR}: 1.81(\mathrm{~s}, 3 \mathrm{H}), 6.17(\mathrm{t}, J=7.3 \mathrm{~Hz}, 1 \mathrm{H})$, $6.46(\mathrm{~d}, J=7.7 \mathrm{~Hz}, 2 \mathrm{H}), 6.62(\mathrm{~m}, 3 \mathrm{H}), 6.77(\mathrm{~m}, 2 \mathrm{H}), 6.88(\mathrm{~d}, J=8.8 \mathrm{~Hz}, 1 \mathrm{H}), 7.06(\mathrm{~m}, 1 \mathrm{H})$, $7.64(\mathrm{~m}, 1 \mathrm{H})$; ${ }^{13} \mathrm{C}-\mathrm{NMR}: 13.04,112.42,114.49,117.42,119.49,122.49,123.58,124.66,126.51$, $126.75,128.60,128.84,133.41,144.69,148.03,154.09$; MS: $m / z(\%) 276\left(\mathrm{M}^{+}, 100\right), 261$ (7), 260 (18), 295 (38), 230 (6), 183 (25), 168 (6), 141 (13), 128 (10), 115 (49), 77 (8); Anal. Calcd. for $\mathrm{C}_{18} \mathrm{H}_{16} \mathrm{~N}_{2} \mathrm{O}: \mathrm{C}, 78.23 ; \mathrm{H}, 5.83 ; \mathrm{N}, 10.13$. Found: C, 77.96; H, 5.71; N, 10.28.

2-Acetyl-1-naphthol 4-chlorophenylhydrazone (2): This compound was prepared from 2-acetyl-1naphthol and 4 -chlorophenylhydrazine using the procedure described for $\mathbf{1}$. Yield, 82\%, brownish solid, $\mathrm{mp} 175^{\circ} \mathrm{C}$; IR: 3,420 (OH), 3,331 (NH), 1,636 (C=N); ${ }^{1} \mathrm{H}-\mathrm{NMR}: 2.38 \quad(\mathrm{~s}, \quad 3 \mathrm{H})$, $6.98(\mathrm{~d}, J=8.8 \mathrm{~Hz}, 2 \mathrm{H}), 7.26(\mathrm{~d}, J=8.8 \mathrm{~Hz}, 2 \mathrm{H}), 7.32(\mathrm{~m}, 2 \mathrm{H}), 7.49(\mathrm{~m}, 2 \mathrm{H}), 7.73(\mathrm{~m}, 1 \mathrm{H}), 8.40$ (m, 1H); ${ }^{13} \mathrm{C}-\mathrm{NMR}: 12.28,112.67,114.27,118.24,123.22,123.51,125.37,125.67,127.15,127.34$, 129.49, 134.28, 142.68, 149.27, 155.67; MS: m/z (\%) $312(\mathrm{M}+2,21), 310\left(\mathrm{M}^{+}, 65\right), 296$ (5), 294 (10), 293 (51), 258 (17), 184 (30), 183 (41), 169 (10), 168 (9), 143 (22), 141 (25), 134 (45), 127 (100), 114 (37), 76 (6); Anal. Calcd. for $\mathrm{C}_{18} \mathrm{H}_{15} \mathrm{ClN}_{2} \mathrm{O}: \mathrm{C}, 69.56$; H, 4.86; N, 9.01. Found: C, 69.72; H, 4.77; 8.88.

2-Acetyl-1-naphthol 4-bromophenylhydrazone (3): This compound was prepared from 2-acetyl-1naphthol and 4̀-bromophenylhydrazine using the procedure described for 1. Yield, 79\%, brownish solid, mp $182^{\circ} \mathrm{C}$; IR: 3,412 (OH), 3,348 (NH), $1,632 \quad(\mathrm{C}=\mathrm{N}) ;{ }^{1} \mathrm{H}-\mathrm{NMR}: 2.40 \quad(\mathrm{~s}, \quad 3 \mathrm{H})$, $6.94(\mathrm{~d}, J=8.1 \mathrm{~Hz}, 2 \mathrm{H}), 7.31(\mathrm{~d}, J=8.8 \mathrm{~Hz}, 1 \mathrm{H}), 7.41(\mathrm{~d}, J=8.8 \mathrm{~Hz}, 1 \mathrm{H}), 7.48(\mathrm{~d}, J=8.1 \mathrm{~Hz}, 2 \mathrm{H})$, $7.53(\mathrm{~m}, 2 \mathrm{H}), 7.74(\mathrm{~m}, 1 \mathrm{H}), 8.41(\mathrm{~m}, 1 \mathrm{H}) ;{ }^{13} \mathrm{C}-\mathrm{NMR}: 12.29,112.92,113.20,118.20,121.12,123.32$, 123.63, 125.33, 125.38, 127.23, 127.30, 129.70, 134.27, 144.11, 148.52, 155.12; MS: m/z (\%) 356 (M+2, 70), $354\left(\mathrm{M}^{+}, 71\right), 340$ (11), 339 (54), 338 (12), 337 (49), 258 (21), 185 (15), 183 (56), 173 (63), 156 (10), 128 (18), 115 (100), 91 (35), 76 (6); Anal. Calcd. for $\mathrm{C}_{18} \mathrm{H}_{15} \mathrm{BrN}_{2} \mathrm{O}$ : C, 60.86; H, 4.25; N, 7.88. Found: C, 60.67; H, 4.15; N, 7.75.

2-Acetyl-1-naphthol 4-methylphenylhydrazone (4): This compound was prepared from 2-acetyl-1naphthol and $\grave{4}$-methylphenylhydrazene using the procedure described for 1. Yield, 91\% brownish solid, mp $164^{\circ} \mathrm{C}$; IR: 3,424 (OH), 3,350 (NH), 1,614 (C=N); ${ }^{1} \mathrm{H}-\mathrm{NMR}: 2.01(\mathrm{~s}, 3 \mathrm{H}), 2.19(\mathrm{~s}, 3 \mathrm{H}), 6.76$ $(\mathrm{d}, J=8.1 \mathrm{~Hz}, 2 \mathrm{H}), 6.80(\mathrm{~d}, J=8.1 \mathrm{~Hz}, 2 \mathrm{H}), 7.02(\mathrm{~m}, 1 \mathrm{H}), 7.17(\mathrm{~m}, 2 \mathrm{H}), 7.24(\mathrm{~m}, 1 \mathrm{H}), 7.45(\mathrm{~m}, 1 \mathrm{H})$, 8.06 (m, 1H); ${ }^{13} \mathrm{C}-\mathrm{NMR}: 12.22,20.73,113.03,113.31,118.13,118.42,123.31,123.62,125.01$, 
125.32, 127.22, 127.49, 130.15, 134.21, 141.96, 148.05, 155.06; MS: m/z (\%) $290\left(\mathrm{M}^{+}, 85\right), 275$ (14), 273 (54), 185 (15), 168 (12), 128 (6), 115 (37), 107 (100), 93 (6), 76 (25); Anal. Calcd. for $\mathrm{C}_{19} \mathrm{H}_{18} \mathrm{~N}_{2} \mathrm{O}: \mathrm{C}, 78.59 ; \mathrm{H}, 7.95 ; \mathrm{N}, 12.27$. Found: C, 78.38; H, 7.85; N, 12.12.

1-Acetyl-2-naphthol phenylhydrazone (5): This compound was prepared from 1-acetyl-2-naphthol and phenylhydrazine hydrochloride using the procedure described for $\mathbf{1}$. Yield, 75\%, pale yellow solid, $\mathrm{mp}$ $125^{\circ} \mathrm{C}$; IR: 3,418 (OH), 3,335 (NH), 1,617 (C=N); ${ }^{1} \mathrm{H}-\mathrm{NMR}: 2.49(\mathrm{~s}, 3 \mathrm{H}), 6.93(\mathrm{t}, J=7.3 \mathrm{~Hz}, 1 \mathrm{H}), 7.12$ $(\mathrm{d}, J=7.3 \mathrm{~Hz}, 2 \mathrm{H}), 7.21(\mathrm{~d}, J=8.8 \mathrm{~Hz}, 1 \mathrm{H}), 7.29(\mathrm{~d}, J=7.3 \mathrm{~Hz}, 2 \mathrm{H}), 7.33(\mathrm{~m}, 1 \mathrm{H}), 7.44(\mathrm{~m}, 1 \mathrm{H}), 7.79$ $(\mathrm{d}, J=8.1 \mathrm{~Hz}, 1 \mathrm{H}) ;{ }^{13} \mathrm{C}-\mathrm{NMR}: 18.41,113.18,116.32,118.64,121.13,123.15,124.52,126.50,128.97$, 129.26, 129.63, 130.77, 131.66, 144.24, 144.76, 153.43; MS: m/z (\%) $276\left(\mathrm{M}^{+}, 69\right), 260$ (21), 259 (100), 184 (22), 169 (6), 141 (9), 128 (7), 115 (28), 93 (33), 77 (9); Anal. Calcd. for $\mathrm{C}_{18} \mathrm{H}_{16} \mathrm{~N}_{2} \mathrm{O}$ : C, 78.23, H, 5.73, N, 10.13. Found: C, 78.11; H, 5.73, N, 10.08 .

1-Acetyl-2-naphthol 4-chlorophenylhydrazone (6): This compound was prepared from 1-acetyl-2naphthol and 4 -chlorophenylhydrazine using the procedure described for 1. Yield, 80\%, pale yellow solid, mp $154^{\circ} \mathrm{C}$; IR: 3,422 (OH), 3,355 (NH), $1,619(\mathrm{C}=\mathrm{N}) ;{ }^{1} \mathrm{H}-\mathrm{NMR}: 2.47(\mathrm{~s}, 3 \mathrm{H}), 7.04$ $(\mathrm{d}, J=9.2 \mathrm{~Hz}, 2 \mathrm{H}), 7.21(\mathrm{~d}, J=9.2 \mathrm{~Hz}, 2 \mathrm{H}), 7.24(\mathrm{~d}, J=8.8,1 \mathrm{H}), 7.33(\mathrm{~m}, 1 \mathrm{H}), 7.44(\mathrm{~m}, 1 \mathrm{H})$, $7.66(\mathrm{~d}, J=8.4 \mathrm{~Hz}, 1 \mathrm{H}), 7.72(\mathrm{~d}, J=8.8 \mathrm{~Hz}, 1 \mathrm{H}), 7.77(\mathrm{~d}, J=7.7 \mathrm{~Hz}, 1 \mathrm{H}) ;{ }^{13} \mathrm{C}-\mathrm{NMR}: 18.42,114.36$, $116.29,118.57,123.24,124.44,126.60,129.00,129.05,129.30,129.51,130.94,131.65,142.93$, 143.11, 153.30; MS: m/z (\%) 312 (M+2, 21), $310\left(\mathrm{M}^{+}, 62\right), 296$ (7), 295 (34), 294 (23), 293 (100), 184 (45), 183 (18), 169 (16), 168 (9), 141 (24), 127 (74), 76 (6); Anal. Calcd. for $\mathrm{C}_{18} \mathrm{H}_{15} \mathrm{ClN}_{2} \mathrm{O}$ : C, 69.56; H, 4.86; N, 9.01. Found: C, 69.42; H, 4.73; N, 9.12.

1-Acetyl-2-naphthol 4-bromophenylhydrazone (7): This compound was prepared from 1-acetyl-2naphthol and 4-bromophenylhydrazine using the procedure described for 1. Yield, 68\%, yellow solid, mp $153^{\circ} \mathrm{C}$; IR: 3,436 (OH), 3,354 (NH), 1,619 (C=N); ${ }^{1} \mathrm{H}-\mathrm{NMR}: 2.49(\mathrm{~s}, 3 \mathrm{H}), 7.01(\mathrm{~d}, J=8.8 \mathrm{~Hz}, 2 \mathrm{H})$, $7.24(\mathrm{~d}, J=8.4 \mathrm{~Hz}, 1 \mathrm{H}), 7.34(\mathrm{~m}, 1 \mathrm{H}), 7.39(\mathrm{~d}, J=8.8 \mathrm{~Hz}, 2 \mathrm{H}), 7.45(\mathrm{~m}, 1 \mathrm{H}), 7.67(\mathrm{~d}, J=8.4 \mathrm{~Hz}, 1 \mathrm{H})$, $7.73(\mathrm{~d}, J=8.8 \mathrm{~Hz}, 1 \mathrm{H}), 7.78(\mathrm{~d}, J=8.4 \mathrm{~Hz}, 1 \mathrm{H})$; ${ }^{13} \mathrm{C}-\mathrm{NMR}: 18.29,112.86,114.66,116.23,118.48$, $123.14,124.35,126.49,128.90,129.20,130.82,131.54,132.31,143.29,145.04,153.19 ; \mathrm{MS}: \mathrm{m} / \mathrm{z}(\%)$ $356(\mathrm{M}+2,71), 354\left(\mathrm{M}^{+}, 75\right), 340$ (19), 339 (97), 338 (22), 337 (100), 258 (8), 210 (17), 185 (12), 183 (27), 173 (44), 169 (67), 115 (87), 76 (7); Anal. Calcd. for $\mathrm{C}_{18} \mathrm{H}_{15} \mathrm{BrN}_{2} \mathrm{O}: \mathrm{C}, 60.86 ; \mathrm{H}, 4.25$; N, 7.88. Found: C, 60.73; H, 4.38; N, 7.93.

1-Acetyl-2-naphthol 4-methylphenylhydrazone (8): This compound was prepared from 1-acetyl-2naphthol and 4-methylphenylhydrazine using the procedure described for 1. Yield, 68\%, yellow solid, mp $132{ }^{\circ} \mathrm{C}$; IR: 3,417 (OH), 3,360 (NH), 1,618 (C=N); ${ }^{1} \mathrm{H}-\mathrm{NMR}: 2.29(\mathrm{~s}, 3 \mathrm{H}), 2.49$ (s, 3H), $7.02(\mathrm{~d}, J=8.1 \mathrm{~Hz}, 2 \mathrm{H}), 7.09(\mathrm{~d}, J=8.1 \mathrm{~Hz}, 2 \mathrm{H}), 7.21(\mathrm{~d}, J=8.8 \mathrm{~Hz}, 1 \mathrm{H}), 7.32(\mathrm{~m}, 1 \mathrm{H}), 7.44(\mathrm{~m}, 1 \mathrm{H})$, $7.68(\mathrm{~d}, J=8.1 \mathrm{~Hz}, 1 \mathrm{H}), 7.72(\mathrm{~d}, J=8.8 \mathrm{~Hz}, 1 \mathrm{H}), 7.78(\mathrm{~d}, J=7.3 \mathrm{~Hz}, 1 \mathrm{H}) ;{ }^{13} \mathrm{C}-\mathrm{NMR}: 18.55,20.69$, $113.38,116.22,118.66,123.15,124.51,126.51,128.96,129.24,130.09,130.62,130.81,131.65$, 142.05, 143.11, 153.54; MS: m/z (\%) $290\left(\mathrm{M}^{+}, 89\right), 273$ (100), 184 (30), 169 (7), 141 (11), 128 (8), 115 (35), 107 (57), 106 (36), 105 (46), 91 (6), 76 (25); Anal. Calcd. for $\mathrm{C}_{19} \mathrm{H}_{18} \mathrm{~N}_{2} \mathrm{O}$ : C, 78.59; H, 7.95; N, 12.27. Found: C, 78.41; H, 7.81; N, 12.18 . 
4-Methylene-3-(N-phenylamino)-3,4-dihydro-2H-naphth[2,1-e]-1,3-oxazine-2-one (9): To a stirred solution of $0.20 \mathrm{~g}(0.72 \mathrm{mmol})$ of 1 and triethylamine $(1 \mathrm{~mL})$ in dichloromethane $(30 \mathrm{~mL})$ was added dropwise under $\mathrm{N}_{2}$ atmosphere a solution of 0.5 equivalent $(0.12 \mathrm{~g}, 0.36 \mathrm{mmol})$ of triphosgene in dichloromethane $(10 \mathrm{~mL})$. The mixture was refluxed for 4 hours and then washed with water. The organic layer was dried over magnesium sulfate and evaporated under vacuum. The resulting solid was recrystallized from a mixture of benzene-ethanol (1:1). Yield, $69 \%$, colorless solid, mp $196^{\circ} \mathrm{C}$; IR: 3,306 (NH), 1,727 (C=O), 1,620 $\left(=\mathrm{CH}_{2}\right)$; ${ }^{1} \mathrm{H}-\mathrm{NMR}: 5.02(\mathrm{~d}, J=2.2 \mathrm{~Hz}, 1 \mathrm{H}), 5.13(\mathrm{~d}, J=2.2 \mathrm{~Hz}, 1 \mathrm{H})$, $6.80(\mathrm{~d}, J=8.1 \mathrm{~Hz}, 2 \mathrm{H}), 7.02(\mathrm{~m}, 3 \mathrm{H}), 7.55(\mathrm{~m}, 2 \mathrm{H}), 7.61(\mathrm{~d}, J=8.8 \mathrm{~Hz}, 1 \mathrm{H}), 7.66(\mathrm{~d}, J=8.8 \mathrm{~Hz}, 1 \mathrm{H})$, $7.83(\mathrm{~m}, 1 \mathrm{H}), 8.20(\mathrm{~m}, 1 \mathrm{H}), 8.33$ (bs, $1 \mathrm{H}) ;{ }^{13} \mathrm{C}-\mathrm{NMR}: 88.60,111.50,113.03,120.69,121.57,122.93$, $125.18,127.46,127.89,128.12,128.60,129.30,134.57,138.69,143.05,146.16,148.05 ; \mathrm{MS}: \mathrm{m} / \mathrm{z}(\%)$ $302\left(\mathrm{M}^{+}, 13\right), 260$ (25), 259 (100), 232 (11), 230 (23), 182 (18), 168 (11), 140 (9), 139 (17), 128 (25), 92 (13), 77 (9); Anal. Calcd. for $\mathrm{C}_{19} \mathrm{H}_{14} \mathrm{~N}_{2} \mathrm{O}_{2}$ : C, 75.48; H, 4.67; N, 9.27. Found: C, 75.13; H, 4.50; $\mathrm{N}, 9.63$.

4-Methylene-3-[N-(4-chlorophenyl)amino]-3,4-dihydro-2H-naphth[2,1-e]-1,3-oxazine-2-one

(10):

Prepared from 2 and triphosgene using the procedure described for 9. Yield, 74\%, pale yellow solid, mp $182^{\circ} \mathrm{C}$; IR: 3,260 (NH), 1,732 (C=O), 1,621 $\left(=\mathrm{CH}_{2}\right)$; ${ }^{1} \mathrm{H}-\mathrm{NMR}: 5.02(\mathrm{~d}, J=2.2 \mathrm{~Hz}, 1 \mathrm{H}), 5.09(\mathrm{~d}, J$ $=2.2 \mathrm{~Hz}, 1 \mathrm{H}), 6.57(\mathrm{bs}, 1 \mathrm{H}), 6.78(\mathrm{~d}, J=9.0 \mathrm{~Hz}, 2 \mathrm{H}), 7.23(\mathrm{~d}, J=9.0 \mathrm{~Hz}, 2 \mathrm{H}), 7.59(\mathrm{~m}, 3 \mathrm{H}), 7.67(\mathrm{~d}$, $J=8.8 \mathrm{~Hz}, 1 \mathrm{H}), 7.85(\mathrm{~m}, 1 \mathrm{H}), 8.30$ (m, 1H); ${ }^{13} \mathrm{C}-\mathrm{NMR}: 88.55,111.09,114.46,115.70,120.24,121.48$, 123.03, 125.36, 127.53, 127.70, 128.22, 132.33, 134.68, 138.26, 143.46, 144.45, 147.99; MS: m/z (\%) 338 (M+2, 6), 336 ( $\left.\mathrm{M}^{+}, 17\right), 301$ (24), 296 (9), 295 (32), 294 (23), 293 (91), 259 (28), 258 (100), 230 (17), 182 (53), 140 (18), 128 (74), 115 (21), 98 (31), 76 (10); Anal. Calcd. for $\mathrm{C}_{19} \mathrm{H}_{13} \mathrm{Cl} \mathrm{N}_{2} \mathrm{O}_{2}$ : C, 67.76; H, 3.89; N, 8.31. Found: C, 67.47; H, 3.71; N, 8.19.

4-Methylene-3-[N-(4-bromophenyl)amino]-3,4-dihydro-2H-naphth[2,1-e]-1,3-oxazine-2-one (11): Obtained from 3 and triphosgene using the procedure described for $\mathbf{9}$. Yield, 71\%, brownish solid, $\mathrm{mp}$ $185^{\circ} \mathrm{C}$; IR: 3,281 (NH), 1,741 $(\mathrm{C}=\mathrm{O}), 1,621\left(=\mathrm{CH}_{2}\right) ;{ }^{1} \mathrm{H}-\mathrm{NMR}: 4.96(\mathrm{~d}, J=2.0 \mathrm{~Hz}, 1 \mathrm{H})$, $5.01(\mathrm{~d}, J=2.0 \mathrm{~Hz}, 1 \mathrm{H}), 6.73(\mathrm{~d}, J=8.8 \mathrm{~Hz}, 2 \mathrm{H}), 7.24(\mathrm{~d}, J=8.8 \mathrm{~Hz}, 2 \mathrm{H}), 7.54(\mathrm{~m}, 4 \mathrm{H}), 7.83(\mathrm{~m}, 1 \mathrm{H})$, 8.17 (m, 1H), 8.69 (bs, 1H); ${ }^{13} \mathrm{C}-\mathrm{NMR}: 88.58,111.33,112.06,114.89,120.73,121.52,122.88,125.27$, 127.53, 127.95, 128.21, 131.99, 134.59, 138.54, 143.35, 145.60, 147.35; MS: m/z (\%) 382 (M+2, 12), 380 ( $\left.\mathrm{M}^{+}, 13\right), 354$ (12), 340 (16), 339 (74), 337 (72), 301 (44), 259 (36), 258 (100), 230 (26), 182 (58), 128 (74), 115 (33), 91 (43), 76 (11); Anal. Calcd. for $\mathrm{C}_{19} \mathrm{H}_{13} \mathrm{BrN}_{2} \mathrm{O}_{2}$ : C, 59.86; H, 3.43; N, 7.34. Found: C, 59.73; H, 3.33; N, 7.43.

4-Methylene-3-[N-(4-methylphenyl)amino]-3,4-dihydro-2H-naphth[2,1-e]-1,3-oxazine-2-one (12): Obtained from 4 and triphosgene using the procedure described for $\mathbf{9}$. Yield, 73\%, brownish solid, mp $150^{\circ} \mathrm{C}$; IR: 3,260 (NH), 1,737 (C=O), 1,612 $\left(=\mathrm{CH}_{2}\right) ;{ }^{1} \mathrm{H}-\mathrm{NMR}: 2.19(\mathrm{~s}, 3 \mathrm{H}), 4.92(\mathrm{~d}, J=2.0 \mathrm{~Hz}, 1 \mathrm{H})$, $5.14(\mathrm{~d}, J=2.0 \mathrm{~Hz}, 1 \mathrm{H}), 6.71(\mathrm{~d}, J=8.1 \mathrm{~Hz}, 2 \mathrm{H}), 7.02(\mathrm{~d}, J=8.1 \mathrm{~Hz}, 2 \mathrm{H}), 7.69(\mathrm{~m}, 2 \mathrm{H})$, $7.84(\mathrm{~d}, J=8.8 \mathrm{~Hz}, 1 \mathrm{H}), 7.91(\mathrm{~d}, J=8.8 \mathrm{~Hz}, 1 \mathrm{H}), 8.04(\mathrm{~m}, 1 \mathrm{H}), 8.17(\mathrm{~m}, 1 \mathrm{H}), 8.67$ (bs, $1 \mathrm{H})$; ${ }^{13}$ C-NMR: $20.72,88.97,111.70,113.07,121.50,122.80,125.43,128.10,128.43,128.62,129.14$, 130.03, 134.66, 138.99, 143.34, 144.14, 147.21; MS: m/z (\%) $316\left(\mathrm{M}^{+}, 24\right), 274$ (20), 273 (100), 244 
(10), 182 (13), 128 (23), 105 (61), 76 (31); Anal. Calcd. for $\mathrm{C}_{20} \mathrm{H}_{16} \mathrm{~N}_{2} \mathrm{O}_{2}$ : C, 75.93; H, 5.10; N, 8.85. Found: C, 75.81; H, 5.15; N, 8.77.

4-Methylene-3-(N-phenylamino)-3,4-dihydro-2H-naphth[1,2-e]-1,3-oxazine-2-one (13): Obtained from 5 and triphosgene using the procedure described for 9 . Yield, $62 \%$, colorless solid, mp $166{ }^{\circ} \mathrm{C}$; IR: 3,297 (NH), 1,725 (C=O), 1,620 $\left(=\mathrm{CH}_{2}\right) ;{ }^{1} \mathrm{H}-\mathrm{NMR}: 5.04(\mathrm{~d}, J=2.2 \mathrm{~Hz}, 1 \mathrm{H}), 5.15(\mathrm{~d}, J=2.2 \mathrm{~Hz}$, $1 \mathrm{H}), 6.53$ (bs, 1H), $6.97(\mathrm{~d}, J=8.8 \mathrm{~Hz}, 2 \mathrm{H}), 7.25(\mathrm{~m}, 3 \mathrm{H}), 7.58(\mathrm{~m}, 3 \mathrm{H}), 7.67(\mathrm{~d}, J=8.1 \mathrm{~Hz}, 1 \mathrm{H}), 7.84$ $(\mathrm{m}, 1 \mathrm{H}), 8.32(\mathrm{~m}, 1 \mathrm{H})$; ${ }^{13} \mathrm{C}-\mathrm{NMR}: 88.53,111.24,113.97,120.33,121.90,122.27,123.10,125.23$, $127.44,127.67,128.12,129.45,134.66,138.46,143.53,145.29,148.07 ; \mathrm{MS}: \mathrm{m} / \mathrm{z}(\%) 302\left(\mathrm{M}^{+}, 7\right)$, 258 (100), 182 (16), 128 (5), 115 (7), 92 (5), 77 (6); Anal. Calcd. for $\mathrm{C}_{19} \mathrm{H}_{14} \mathrm{~N}_{2} \mathrm{O}_{2}$ : C, 75.48; H, 4.67; N, 9.27. Found: C, 75.24; H, 4.71; N, 9.18.

4-Methylene-3-[N-(4-chlorophenyl)amino]-3,4-dihydro-2H-naphth[1,2-e]-1,3-oxazine-2-one (14):

Obtained from 6 and triphosgene using the procedure described for 9. Yield, 61\%, brownish solid, $\mathrm{mp}$ $187^{\circ} \mathrm{C}$; IR: 3,260 (NH), 1,732 $(\mathrm{C}=\mathrm{O}), 1,620\left(=\mathrm{CH}_{2}\right) ;{ }^{1} \mathrm{H}-\mathrm{NMR}: 5.05(\mathrm{~d}, J=1.5 \mathrm{~Hz}, 1 \mathrm{H})$, $5.15(\mathrm{~d}, J=1.5 \mathrm{~Hz}, 1 \mathrm{H}), 6.52(\mathrm{bs}, 1 \mathrm{H}), 6.84(\mathrm{~d}, J=8.8 \mathrm{~Hz}, 2 \mathrm{H}), 7.23(\mathrm{~d}, J=8.8 \mathrm{~Hz}, 2 \mathrm{H})$, $7.51(\mathrm{~m}, 3 \mathrm{H}), 7.70(\mathrm{~d}, J=8.1 \mathrm{~Hz}, 1 \mathrm{H}), 7.86(\mathrm{~m}, 1 \mathrm{H}), 8.31(\mathrm{~m}, 1 \mathrm{H}) ;{ }^{13} \mathrm{C}-\mathrm{NMR}: 88.53,111.10,115.33$, $120.25,121.87,123.04,125.37,127.22,127.54,127.71,128.23,129.44,134.69,138.30,143.48$, 143.95, 148.01; MS: m/z (\%) $338(\mathrm{M}+2,5), 336\left(\mathrm{M}^{+}, 14\right), 301$ (24), 295 (29), 259 (29), 258 (100), 230 (14), 182 (60), 128 (68), 115 (21), 99 (36), 76 (9); Anal. Calcd. for $\mathrm{C}_{19} \mathrm{H}_{13} \mathrm{Cl} \mathrm{N}_{2} \mathrm{O}_{2}$ : C, 67.76; H, 3.89; N, 8.32. Found: C, 67.59; H, 3.95; N, 8.25.

4-Methylene-3-[N-(4-bromophenyl)amino]-3,4-dihydro-2H-naphth[1,2-e]-1,3-oxazine-2-one (15): Obtained from 7 and triphosgene using the procedure described for 9. Yield, 64\%, colorless solid, $\mathrm{mp}$ $203^{\circ} \mathrm{C}$; IR: $3,353(\mathrm{NH}), 1,733(\mathrm{C}=\mathrm{O}), 1,616\left(=\mathrm{CH}_{2}\right) ;{ }^{1} \mathrm{H}-\mathrm{NMR}: 5.05(\mathrm{~d}, J=2.2 \mathrm{~Hz}, 1 \mathrm{H})$, $5.14(\mathrm{~d}, J=2.2 \mathrm{~Hz}, 1 \mathrm{H}), 6.53(\mathrm{bs}, 1 \mathrm{H}), 6.90(\mathrm{~d}, J=8.0 \mathrm{~Hz}, 2 \mathrm{H}), 7.29$ (d, $J=8.0 \mathrm{~Hz}, 2 \mathrm{H}), 7.60$ (m, $3 \mathrm{H}), 7.72(\mathrm{~d}, J=8.8 \mathrm{~Hz}, 1 \mathrm{H}), 7.82(\mathrm{~m}, 1 \mathrm{H}), 8.12(\mathrm{~m}, 1 \mathrm{H}) ;{ }^{13} \mathrm{C}-\mathrm{NMR}: 88.54,111.24,113.96,116.78$, 120.33, 121.90, 125.24, 127.16, 127.45, 127.68, 128.13, 129.47, 134.66, 138.45, 143.53, 145.29, 148.08; MS: m/z (\%) 382 (M+2, 18), $380\left(\mathrm{M}^{+}, 16\right), 301$ (35), 259 (30), 258 (100), 230 (35), 210 (18), 184 (49), 182 (48), 139 (45), 126 (69), 115 (72), 101 (35), 76 (19); Anal. Calcd. for $\mathrm{C}_{19} \mathrm{H}_{13} \mathrm{BrN}_{2} \mathrm{O}_{2}$ : C, 59.86; H, 3.44; N, 7.35. Found: C, 59.98; H, 3.18; N, 7.12.

4-Methylene-3-[N-(4-methylphenyl)amino]-3,4-dihydro-2H-naphth[1,2-e]-1,3-oxazine-2-one

(16):

Obtained from 8 and triphosgene using the procedure described for $\mathbf{9}$. Yield $60 \%$, yellowish solid, $\mathrm{mp}$ $160^{\circ} \mathrm{C}$; IR: 3,245 (NH), 1,741 (C=O), 1,615 $\left(=\mathrm{CH}_{2}\right) ;{ }^{1} \mathrm{H}-\mathrm{NMR}: 2.26(\mathrm{~s}, 3 \mathrm{H}), 5.04(\mathrm{~d}, J=2.20 \mathrm{~Hz}, 1 \mathrm{H})$, $5.14(\mathrm{~d}, J=2.20 \mathrm{~Hz}, 1 \mathrm{H}), 6.47(\mathrm{bs}, 1 \mathrm{H}), 6.81(\mathrm{~d}, J=8.8 \mathrm{~Hz}, 2 \mathrm{H}), 7.08(\mathrm{~d}, J=8.8 \mathrm{~Hz}, 2 \mathrm{H}), 7.58(\mathrm{~m}$, 3H), $7.66(\mathrm{~d}, J=8.8 \mathrm{~Hz}, 1 \mathrm{H}), 7.85(\mathrm{~m}, 1 \mathrm{H}), 8.32(\mathrm{~m}, 1 \mathrm{H}) ;{ }^{13} \mathrm{C}-\mathrm{NMR}: 20.71,88.50,111.28,114.12$, $120.34,121.91,123.10,125.19,127.43,127.67,128.10,129.96,131.73,134.63,138.48,142.88$, 143.53, 148.10; MS: m/z (\%) $316\left(\mathrm{M}^{+}, 13\right) 274$ (17), 273 (100), 182 (11), 139 (15), 128 (20), 106 (52), 76 (36); Anal. Calcd. for $\mathrm{C}_{20} \mathrm{H}_{16} \mathrm{~N}_{2} \mathrm{O}_{2}$ : C, 75.93; H, 5.10; N, 8.85. Found: C, 75.75; H, 5.22; N, 8.73. 
3 '-Phenyl-3-(phenylamino)-1',12 'b-dihydro-12 'b-methylspiro $44 H$-naphth [2,1-e]-1,3-oxazine-4,2' (3'H)- [5H]pyrazolo[1,5-c]naphth[2,1-e]-1,3-oxazine\}-2,5'-dione (17): Obtained from 1 and one equivalent of triphosgene using the procedure described for 9. Yield $69 \%$, reddish solid, mp $207{ }^{\circ} \mathrm{C}$; IR: 3,256 (NH), 1,748, 1,755 (2C=O); ${ }^{1} \mathrm{H}-\mathrm{NMR}: 2.12(\mathrm{~s}, 3 \mathrm{H}), 3.58(\mathrm{~d}, J=14.70 \mathrm{~Hz}, 1 \mathrm{H})$, $3.65(\mathrm{~d}, J=14.70 \mathrm{~Hz}, 1 \mathrm{H}), 6.72(\mathrm{~m}, 4 \mathrm{H}), 6.97(\mathrm{~m}, 4 \mathrm{H}), 7.11(\mathrm{~m}, 5 \mathrm{H}), 7.58(\mathrm{~m}, 5 \mathrm{H}), 7.91(\mathrm{~m}, 2 \mathrm{H}), 8.40$ (m, 2H); ${ }^{13} \mathrm{C}-\mathrm{NMR}: 32.15,59.65,66.06,85.55,112.48,112.93,113.91,116.47,116.80,119.92$, $120.36,121.68,122.19,122.23,124.96,125.49$, 127.55, 127.68, 128.81, 129.19, 133.56, 133.86, $134.09,141.92,142.46,142.78,143.18,145.29,145.42,145.69,149.24,149.90 ; \mathrm{MS}: \mathrm{m} / \mathrm{z}(\%) 471$ $\left(\mathrm{M}^{+}-\mathrm{C}_{6} \mathrm{H}_{5} \mathrm{~N}_{2} \mathrm{CO}, 20\right), 455\left(\mathrm{M}^{+}-\mathrm{C}_{6} \mathrm{H}_{5} \mathrm{~N}_{2} \mathrm{CO}_{2}, 14\right), 440$ (57), 423 (28), 351 (12), 302 (21), 259 (100), 258 (17), 230 (34), 185 (29), 168 (39), 128 (21), 115 (38), 93 (70), 77 (51), 63 (35); Anal. Calcd. for $\mathrm{C}_{38} \mathrm{H}_{28} \mathrm{~N}_{4} \mathrm{O}_{4}$ : C, 75.48; H, 4.66; N, 9.26. Found: C, 75.21; H, 4.71; N, 9.19.

3'-(4-Chlorophenyl)-3-[(4-chlorophenyl)amino]-1',12'b-dihydro-12'-b-methylspiro 4 4H-naphth[1,2e]-1,3-oxazine-4,2'(3'H)-[5H]pyrazolo[1,5-c]naphth[2,1-e]-1,3-oxazine\}-2,5'-dione (18): This compound was obtained from 2 and one equivalent of triphosgene using the procedure described for 9. Yield, $72 \%$, mp $267^{\circ} \mathrm{C}$; IR: 3,219 (NH), 1,724, $1,753 \quad(2 \mathrm{C}=\mathrm{O})$; ${ }^{1} \mathrm{H}-\mathrm{NMR}: 2.10 \quad(\mathrm{~s}, \quad 3 \mathrm{H})$, $3.49(\mathrm{~d}, J=14.70 \mathrm{~Hz}, 1 \mathrm{H}), 3.60(\mathrm{~d}, J=14.70 \mathrm{~Hz}, 1 \mathrm{H}), 6.35(\mathrm{~m}, 3 \mathrm{H}), 6.65(\mathrm{~m}, 4 \mathrm{H}), 6.93(\mathrm{~m}, 4 \mathrm{H}), 7.66$ $(\mathrm{m}, 5 \mathrm{H}), 7.82(\mathrm{~m}, 2 \mathrm{H}), 8.40(\mathrm{~m}, 2 \mathrm{H}) ;{ }^{13} \mathrm{C}-\mathrm{NMR}: 32.08,60.07,66.05,85.47,111.88,114.22,117.91$, $119.62,121.02,121.54,122.16,122.56,123.11,125.25,125.57,126.45,127.18,127.40,127.47$, $127.66,127.78,127.93,128.81,128.84,128.91,133.47,134.31,141.39,141.81,143.90,145.61$, 149.61, 149.01, 149.07; MS: m/z (\%) $505\left(\mathrm{M}^{+}-\mathrm{ClC}_{6} \mathrm{H}_{4} \mathrm{~N}_{2} \mathrm{CO}, 22\right), 489\left(\mathrm{M}^{+}-\mathrm{ClC}_{6} \mathrm{H}_{4} \mathrm{~N}_{2} \mathrm{CO}_{2}, 100\right)$, 410 (7), 336 (13), 295 (12), 259 (21), 230 (10), 168 (26), 139 (24), 127 (28), 115 (21), 99 (9), 90 (5), 76 (15), 63 (12); Anal. Calcd. for $\mathrm{C}_{38} \mathrm{H}_{26} \mathrm{Cl}_{2} \mathrm{~N}_{4} \mathrm{O}_{4}$ : C, 67.76; H, 3.89; N, 8.32. Found: C, 67.46; H, $3.68 ; \mathrm{N}, 8.54$.

3'-(4-Bromophenyl)-3-[(4-bromophenyl)amino]-1',12'b-dihydro-12 'b-methylspiro 44H-naphth[2,1-e]1,3-oxazine-4,2'(3'H)-[5H]pyrazolo[1,5-e]naphth [2,1-e]-1,3-oxazine\}-2,5'-dione

(19): Obtained from 3 and one equivalent of triphosgene using the procedure described for 9 . Yield, $71 \%, \mathrm{mp} 246{ }^{\circ} \mathrm{C}$; IR: 3,232 (NH), 1,730, 1,745 (2C=O); ${ }^{1} \mathrm{H}-\mathrm{NMR}: 2.09(\mathrm{~s}, 3 \mathrm{H}), 3.44(\mathrm{~d}, J=14.70 \mathrm{~Hz}, 1 \mathrm{H})$, $3.54(\mathrm{~d}, J=14.70 \mathrm{~Hz}, 1 \mathrm{H}), 6.28(\mathrm{~m}, 3 \mathrm{H}), 6.60(\mathrm{~m}, 3 \mathrm{H}), 6.85(\mathrm{~m}, 2 \mathrm{H}), 7.04(\mathrm{~m}, 2 \mathrm{H}), 7.52(\mathrm{~m}, 6 \mathrm{H})$, $7.78(\mathrm{~m}, 2 \mathrm{H}), 8.34(\mathrm{~m}, 2 \mathrm{H}) ;{ }^{13} \mathrm{C}-\mathrm{NMR}: 32.04,60.11,65.96,85.41,111.75,113.97,114.67,115.87$, $117.98,118.21,119.56,121.00,121.53,122.16,122.55,123.11,125.31,127.42,127.48,127.69$, 127.79, 127.95, 128.94, 131.70, 131.74, 132.05, 133.66, 134.31, 141.78, 141.88, 144.40, 148.95, 149.15; MS: m/z (\%) $549\left(\mathrm{M}^{+}-\mathrm{BrC}_{6} \mathrm{H}_{4} \mathrm{~N}_{2} \mathrm{CO}, 6\right), 533\left(\mathrm{M}^{+}-\mathrm{BrC}_{6} \mathrm{H}_{4} \mathrm{~N}_{2} \mathrm{CO}_{2}, 100\right), 409$ (15), 382 (20), 380 (17), 340 (24), 339 (85), 258 (86), 202 (24), 196 (51), 168 (74), 129 (59), 115 (84), 99 (51), 91 (49), 90 (52), 76 (71), 63 (84); Anal. Calcd. for $\mathrm{C}_{38} \mathrm{H}_{26} \mathrm{Br}_{2} \mathrm{~N}_{4} \mathrm{O}_{4}$ : C, 59.86; H, 3.43; N, 7.34. Found: C, $59.73 ; \mathrm{H}, 3.49 ; \mathrm{N}, 7.42$.

3'-(4-Chlorophenyl)-3-[(4-chlorophenyl)amino]-1',12 'b-dihydro-12'b-methylspiro\{4H-naphth [1,2-e]1,3-oxazine-4,2'(3'H)-[5H]pyrazolo[1,54-c]naphth[1,2-e]-1,3-oxazine\}-2,5'-dione (20): Obtained from 6 and one equivalent of triphosgene using the procedure described for $\mathbf{9}$. Yield, 55\%; mp $227{ }^{\circ} \mathrm{C}$; IR: 3,338 (NH), 1,730, 1,755 (2C=O); ${ }^{1} \mathrm{H}-\mathrm{NMR}: 2.51(\mathrm{~s}, 3 \mathrm{H}), 3.41(\mathrm{~d}, J=14.65 \mathrm{~Hz}, 1 \mathrm{H})$, 
$3.54(\mathrm{~d}, J=14.65 \mathrm{~Hz}, 1 \mathrm{H}), 7.07(\mathrm{~m}, 3 \mathrm{H}), 7.25(\mathrm{~m}, 4 \mathrm{H}), 7.51(\mathrm{~m}, 5 \mathrm{H}), 8.11(\mathrm{~m}, 4 \mathrm{H}), 8.29(\mathrm{~m}, 2 \mathrm{H})$, 8.61 (m, 2H); ${ }^{13} \mathrm{C}-\mathrm{NMR}: 17.20,27.87,88.06,97.68,114.00,114.31,114.46,115.89,116.26,120.55$, $123.56,124.20,124.87,125.67,125.73,127.89,128.57,128.72,128.87,128.93,129.76,131.21$, 131.32, 131.66, 131.77, 131.80, 136.60, 138.88, 143.81, 144.17, 145.50, 147.04, 148.60, 149.01; MS: $\mathrm{m} / \mathrm{z}(\%) 505\left(\mathrm{M}^{+}-\mathrm{ClC}_{6} \mathrm{H}_{4} \mathrm{~N}_{2} \mathrm{CO}, 20\right), 489\left(\mathrm{M}^{+}-\mathrm{ClC}_{6} \mathrm{H}_{4} \mathrm{~N}_{2} \mathrm{CO}_{2}, 100\right), 410$ (7), 336 (21), 319 (15), 295 (21), 293 (44), 258 (31), 230 (14), 139 (29), 127 (44), 115 (35), 75 (20), 63 (16); Anal. Calcd. for $\mathrm{C}_{38} \mathrm{H}_{26} \mathrm{Cl}_{2} \mathrm{~N}_{4} \mathrm{O}_{4}$ : C, 67.76; H, 3.89; N, 8.32. Found: C, 67.53; H, 3.93; N, 8.46.

\section{References}

1. Di Santo, R.; Costi, R.; Artico, M.; Massa, S.; Morangiu, M.E.; Loi, A.G.; De Montis, A.; La Colla, P. 1,2,5-Benzothiadiazepine and pyrrolo[2,1d][1,2,5]benzothiadiazepine derivatives with specific anti-human immunodeficiency virus type 1 activity. Antiviral Chem. Chemother. 1998, 9 , 127.

2. Chung, Y.J.; Jung, Y.S.; Seong, C.M.; Park, N.S. An Efficient Synthesis of 3-Substituted Quinazolones. Bull. Korean Chem. Soc. 1998, 19, 1117.

3. Nefzi, A.; Otresh, J.M.; Giulianotti, M.; Hougten, R.A. Efficient solid phase synthesis of 3,5disubstituted hydantoins. Tetrahedron Lett. 1998, 39, 8199.

4. Li, Z.; Zhang, Y. Low-valent titanium induced cyclization of dimeric dianions of anils with triphosgene: a safe and efficient method for the syntheses of substituted imidazolidine 2-ones. Synth. Commun. 2002, 32, 2613.

5. Krishnaswamy, D.; Govande,V.V.; Gumaste, V.K.; Bhawal, B.M.; Deshmukh, A.R.A.S. Triphosgene: a versatile reagent for the synthesis of azetidin-2-ones. Tetrahedron 2002, 58, 2215.

6. Alkhathlan, H.Z. Synthesis of 4-alkoxy-4-methyl- and 4-alkoxy-4-fluoromethyl-1,3benzoxazinones. Tetrahedron 2003, 59, 8163.

7. Alkhathlan, H.Z. A One-Pot Synthesis of 1,3-Benzoxazines. Synth. Commun. 2004, 34, 71.

8. Alkhathlan, H.Z.; Al-Saad, M.A.; Al-Hazimi, H.M.; Al-Farhan, K.A.; Mousa, A.A. Synthesis and X-ray crystal structures of novel spiro 1,3-benzoxazine dimmers. J. Chem. Res. 2002, (S) 473, (M) 1023.

9. Alkhathlan, H.Z.; Al-Saad, M.A.; Al-Hazimi, H.M.; Al-Farhan, K.A.; Mousa, A.A. Quinazoline, pyrazolo[1,5-c]quinazoline and spiro quinazoline dimers from the reaction of 2aminoacetophenone hydrazones with triphosgene. J. Chem. Res. 2002, (S) 587, (M) 1201.

10. Molina, P.; Conesa, C.; Alias, A.; Arques, A.; Velasco, M. Preparation and synthetic applications of iminophosphoranes derived from $o$-substituted arylazides: preparation of pyrazolo [1,2-b] indazole, 4H-3,1-benzoxazine and quinoline derivatives. Crystal structure of 2-[2-(4-methoxybenzoylamino)phenyl]-4-methylquinoline. Tetrahedron 1993, 49, 7599.

Sample Availability: Samples of the compounds are available from the authors.

(C) 2009 by the authors; licensee Molecular Diversity Preservation International, Basel, Switzerland. This article is an open-access article distributed under the terms and conditions of the Creative Commons Attribution license (http://creativecommons.org/licenses/by/3.0/). 